\title{
Anti-inflammatory role of omega-3 to retinal injury induced by infrared radiation
}

\author{
Mervat Ahmed Ali \\ Research institute of ophthalmology, Visual science department, Giza, Egypt
}

Email address:

Mervat_galal18@yahoo.com (M. Ahmed Ali)

\section{To cite this article}

Mervat Ahmed Ali. Anti-Inflammatory Role of Omega-3 to Retinal Injury induced by Infrared Radiation. American Journal of Life Sciences. Vol. 1, No. 2, 2013, pp. 49-54. doi: 10.11648/j.ajls.20130102.15

\begin{abstract}
Each ocular tissue is vulnerable to infrared (IR) since at every stage through the eye's transmission IR is absorbed. Thermal effect of IR to eyes is far more permanent although the absorption often causes cumulative damage over a long period. Nevertheless, those exposed to very significant level of IR at any one time would be aware of its heating effect and tend to protect themselves. The present work investigated the effect of IR on retina and the anti-inflammatory role of omega-3 polyunsaturated fatty acid ( $\omega-3$ PUSFA). The rabbits were divided into four groups; one of them served as control, the other three groups was exposed to IR for 5, 10 and 20 minutes. Animals from these three groups were subdivided into two subgroups, one of them was sacrificed directly after IR exposure, while the other received omega-3 for 14 days before exposed to IR .The animals were subjected to examination by electroretinogram (ERG) as well as analysis of Sodium dodecyl sulfate polyacrylamide gel electrophoresis (SDS-PAGE) for retinal protein was carried out. The results indicated decrease of b-wave amplitude and increase in the latency, in the electrophoresis pattern pronounced changes were observed. These changes were more extensive in rabbits exposed to IR than those supplemented with omega-3. These finding suggest that omega-3 can protect the retina from infrared injury for up to 10 minutes of exposure.
\end{abstract}

Keywords: Infrared radiation, Omega-3, Electroretinogram, rabbits, Electrophoresis

\section{Introduction}

Infrared radiation (IR) is that part of the electromagnetic spectrum associated with energy levels such that thermal effects are produced when it is absorbed by matter. All sources can be classified as either artificial or natural (sun) and most sources that emit ultraviolet or visible radiation will probably emit IR. This is important in considering the potential occupational hazards from the multitude of artificial radiation sources. The infrared region exists between the visible and microwave subdivisions of the electromagnetic spectrum and is divided into the following three, biologically significant, bands: IR-A (near IR) between 760 and $1400 \mathrm{~nm}$, IR-B (mid IR) between 1400 and $3000 \mathrm{~nm}$, and IR-C (far IR) between 3000 and $10^{6} \mathrm{~nm}$ [1]. IR-A radiation specifically is absorbed by the retina and is very ineffective in producing retinal injuries [2]. In the occupational setting, any work involving high temperature furnaces such as glass-making or the iron and steel industries are areas where risk assessments for IR exposure to eyes needs to be taken seriously. Many lasers emit IR, in particular ones used in medical fields such as the neodymium YAG laser emitting in the IR-A bands and the C [O.sub.2] laser, which emits in the IR-C range. Arc lamps and any radiant heater also emit IR. Retinal burns from exposure to industrial sources, such as xenon lamps, infrared lasers and metal arc inert gas welding, are typical. Deliberate gazing at the sun has been show to produce a solar retinopathy, on account of the thermal damage and is typical to that seen in viewing solar eclipses. These effects are normally permanent [3-6]. It is generally assumed that IR photons, because of their low energy levels, do not react photochemical in biological tissues [7] but the synergism between thermal and photochemical effects in the lens and retina has been studied in some experiments. Thermal enhancement of photochemical reaction has been experimentally demonstrated [8,9], although the effect is less than a factor of two; this has been taken into account. Many studies have attempted to establish threshold exposure values for the human retina but this is very complex area. In addition to the physical factors, such as exposure duration and irradiance levels, eyes vary in their degree of retinal pigmentation, which undoubtedly has an effect on the degree and extent of damage by infrared radiation. Studies have shown that the longer wavelength infrared radiation requires 
higher irradiance at the cornea to produce a retinal burn. This is pertinent since at lower wavelengths, much more infrared passes through to the retina [10]. Work on rhesus monkeys in 1979 by Ham et al [11] found that to produce retinal lesions of $159 \mu \mathrm{m}$ in size (which is the size of the sun's image produced on the retina by sun gazing), radiant exposures on the retina from two spectral wavebands (400-800 $\mathrm{nm}$ and 700-1400 nm) were required with exposure durations from 1-1000 seconds. The inverse relationship between power and duration was maintained for exposure durations of 10 seconds or longer, with the radiant exposure (product of power and duration) being $400 \mathrm{Jcm}$ for the 400- $800 \mathrm{~nm}$ waveband. For the $700-1400 \mathrm{~nm}$ waveband, a radiant exposure of $70000 \mathrm{Jcm}$ was required, with duration of 1000 seconds only, since they were unable to produce a retinal burn with exposures shorter than 1000 seconds. In another study in which the heat transport within both the human and rabbit's eyes was calculated, the calculated ocular temperature was rapidly increasing with the exposure time for the first 2 minutes, then gradually leveled off and reached the maximum within approximately 5 minutes. Furthermore, it takes several minutes for the eye to cool down after the exposure ceases [12, 13].The Security Equipment Assessment Laboratory on the hazards of IR when installing surveillance features utilizing IR emitters found that the minimum safe exposure for IR at $10 \mathrm{~mW} / \mathrm{cm}^{2}$ was 1000 seconds of continuous exposure before there was a risk of eye damage [14].

Omega-3 fatty acids (also called $\omega^{-3}$ fatty acids or $n-3$ fatty acids) are fats commonly found in marine and plant oils. They are long chain polyunsaturated fatty acids (LCPUFAs) with a double bond $(\mathrm{C}=\mathrm{C})$ starting after the third carbon atom from the end of the carbon chain. Mammals depend on dietary intake of n-3 fatty acids through sources such as fish oil, because mammalian cells lack enzymes necessary to synthesize the 18-C precursor of n-3 fatty acids and to convert n-6 to n-3 fatty acids [15] n-3 fatty acids are highly concentrated in the brain and retina and are believed to be important in neuronal development and damage repair[16]. Docosahexaenoic acid (DHA) and eicosapentaenoic acid (EPA, the precursor to DHA) are two major n-3 fatty acids and are concentrated in retina and retinal vascular endothelium [17]. Photoreceptors are abundantly enriched in DHA with amounts up to approximately $50 \%$ of photoreceptor rod outer segment lipids [18]. Vital retinal functions depend on the existence of an adequate proportion of DHA in retinal lipids [19]. DHA prevents the apoptosis of photoreceptor cells that otherwise inevitably occurs during their early development in vitro [20]. Biophysical and biochemical properties of DHA may affect photoreceptor membrane function by altering permeability, fluidity, thickness, and lipid phase properties. Tissue DHA status affects retinal cell signaling mechanisms involved in phototransduction. DHA may operate in signaling cascades to enhance activation of membrane-bound retinal proteins and may also be involved in rhodopsin regeneration. Tissue DHA insufficiency is associated with alterations in retinal function. Omega-3 ( $\omega-3)$
(LCPUFAs) exhibit cytoprotective and cytotherapeutic actions contributing to a number of anti-angiogenic and neuroprotective mechanisms within the retina. $\omega-3$ LCPUFAs may modulate metabolic processes and attenuate effects of environmental exposures that activate molecules implicated in pathogenesis of vasoproliferative and neurodegenerative retinal diseases. These processes and exposures include ischemia, chronic light exposure, oxidative stress, inflammation, cellular signaling mechanisms, and aging [17].

The present study investigated the effect of irradiation to IR with different exposure times (5, 10 and 20 minutes) and the role of omega-3 in altered retinal function as assessed by SDS polyacrylamide gel electrophoresis and electroretinogram.

\section{Materials and Methods}

Sixteen healthy New Zealand, albino rabbits of either sex, weighing $2-2.5 \mathrm{~kg}$, were used in this study. Animals were randomly selected from the animal house facility at the Research Institute of Ophthalmology, Giza, Egypt. The experimental protocol was approved by the local ethical committee that applies ARVO (The Association for Research in Vision and Ophthalmology) statements of using animals in ophthalmic and vision research. Rabbits were divided into four groups. One of them served as control, the other three groups were exposed to IR for 5, 10 and $20 \mathrm{mi}$ nutes. Animals from these three groups were subdivided into two subgroups. One of them was decapitated directly after IR exposure, while the other received omega -3 for 14 days before exposed to IR.

Omega -3 (Omega 300 Arab Co. of gelatinous, pharmaceutical products, Egypt) used in this study was in the form of fish-oil, gelatinous capsules, containing $1000 \mathrm{mg}$ fish-oil. Each capsule was evacuated by syringe carefully and given to rabbits at a dose of $300 \mathrm{mg} / \mathrm{kg}$ once a day by an intra-gastric tube.

\subsection{Source}

IR was delivered from a General Electric Lamp, model 250R 50/10, placed $20 \mathrm{~cm}$ from the rabbit and aimed at each eye. The lamp was calibrated at the Photometry Department, National Institute of standards, Giza, Egypt. The wavelengths emitted from the anterior surface of the IR lamp as provided by General Electric Lighting Division (Cleveland, OH, USA)[21] were 0.34-0.4 $\mu \mathrm{m}$ (UV light), 0.4-0.76 $\mu \mathrm{m}$ (visible light), 0.76-3.0 $\mu \mathrm{m}$ (IR-A, IR-B light 83\%) and $3.0-7.0 \mu \mathrm{m}$ (IR-C 10\%). The total IR percentage emitted was $93 \%$. The irradiance of the IR lamp detected at $20 \mathrm{~cm}$ was $0.2 \mathrm{~W} / \mathrm{cm} 2$.

\subsection{Electroretinogram}

The animals were dark adapted for 3 hours before the electrophysiological recording. They were anesthetized intraperitoneally first with $100 \mathrm{mg} / \mathrm{Kg}$ ketamine then 5 $\mathrm{mg} / \mathrm{kg}$ xylazine. After establishing the anesthesia, animals 
were placed on the pad of an operating table where their body temperature was maintained at $37^{\circ} \mathrm{C}$. Each rabbit was positioned with its head resting to one side and local anesthetizing eye drops were also applied. The electroretinogram (ERG) was recorded by using three $\mathrm{Ag}-\mathrm{AgCl}$ electrodes. The active electrode was a wick electrode placed at the corneal periphery. The other two electrodes were placed on the skin of the lower eyelid and on the ear, as reference and earthed electrodes, respectively. A white flash was used in this work with fixed intensity (4 lux) and duration (0.2 s). The resulted electrophysiological signals were pre-amplified using CEPTU pream. (Bioscience, UK). The pre-amplified signals were delivered to a computer system attached to a digital oscilloscope (Velleman Co., Taiwan), to be recorded and analyzed by the provided software.

\subsection{Sodium Dodecyl Polyacrylamides-Gel Electrophe- $\operatorname{sis}(S D S-P A G E)$}

After electroretinogram, rabbits from both control and experimental groups were sacrificed and biopsies of retina were taken and processed for SDS -PAGE according to laemmli 1970 [22]. Electrophoresis was carried out with constant volt at $200 \mathrm{~V}$, using 5\% stacking gel and 12\% separating gel. The separated proteins on polyacrylamides gel were stained with coomassie blue R-250. The data were representing graphically with an automatic scanner (model R-112, manufactured by Beckman coulter, CA, USA).

\subsection{Statistical Analysis}

Data were presented as the mean $\pm \mathrm{SD}$. To determine the significant difference between the groups, analysis of variance (ANOVA) procedure was used followed by student's t-test, where commercially available statistical software package, SPSS-11 for windows, was used. The significance level was set at $\mathrm{P}<0.05$

\section{Results}

The flash electroretinogram (ERG) is a valuable tool for assessing retinal function as shown in figure (1) which illustrated a typical ERG after dark adaptation and is characterized by a prominent positive going $b$ - wave arising in the inner unclear layer.

Figures (2) and (3) illustrated b-wave amplitude and latency respectively as a function of the time of exposure to IR without or with supplementation of omega-3. After $5 \mathrm{~min}$ exposure, there was slightly reduction in the amplitude wave by $8 \%$ and the latency increase by $-2 \%$. But for animals supplemented with omega-3, there was no significant difference formed when compared with the control group. After a 10 min exposure to IR without or with omega-3 supplementation, the marked reduction of $b$-wave amplitude by $72 \%$ and $60 \%$ and latency increase by $-6 \%$ and $-4 \%$ respectively. It was clear there was a significant improvement in b-wave in rabbits treated with omega- 3 as compared with untreated but amplitude and latency of b-wave didn't return to normal value. By increasing the time of exposure to IR for $20 \mathrm{~min}$, there was no significant difference in rabbits supplemented without or with omega-3, a selective reduction of the b-wave was observed to the extent that the peak of b-wave failed to reach the baseline and substantially increased noise level.
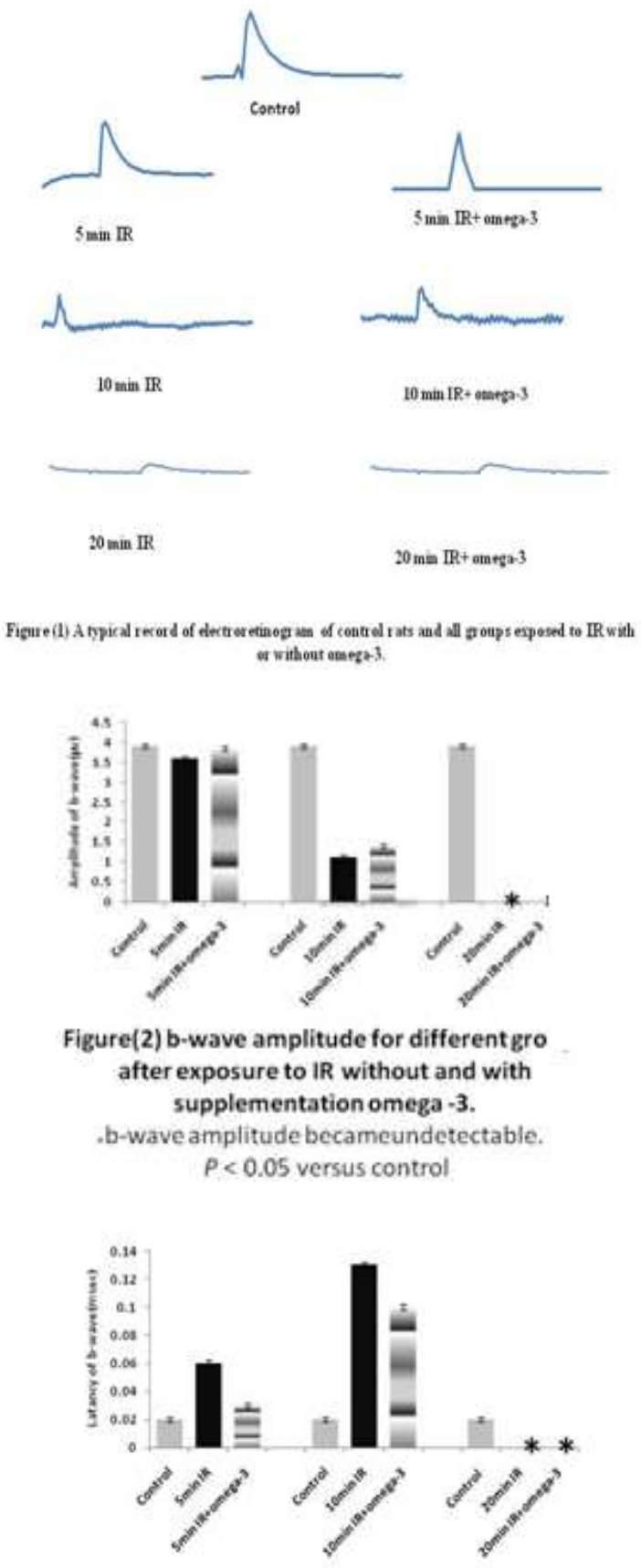

Figure(3) Latency of b-wave for different groups aft $\epsilon$ exposure to IR without and with supplementation omega-3.

Latency of b-wave amplitude became undetectable $P<0.05$ versus control

Figure (4) illustrates the electrophoresis pattern of normal rabbit's retina exposed to infrared (IR) radiation for $5 \mathrm{mi}$ nutes and retina from rabbits supplemented with omega -3 two weeks before exposure to IR. The normal pattern was 
characterized by 8 fractions represent the different proteins from retina with a molecular weights range of (145-10 KDa). After exposure to 5 minutes, the intensity of different bands significantly decreased and the peaks were shifted towards low molecular weight. After supplementation with omega-3, the electrophoretic pattern, to some extent, was match the control.

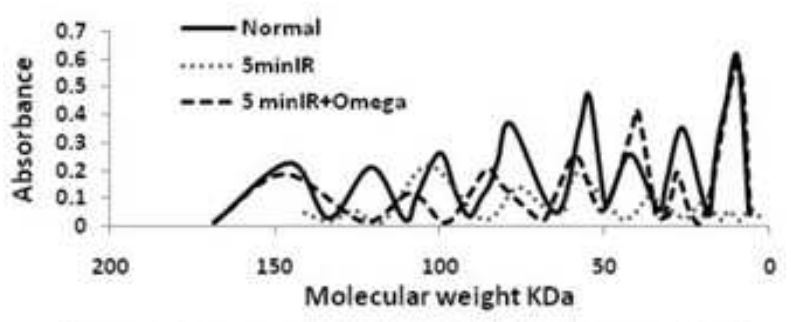

Figure (4):Flectrophoretic pattern of rat's retina for normal and exposed to IR for $5 \mathrm{~min}$ with and without ornega-3.

After exposure to IR for 10 minutes the electrophoretic pattern was shifted towards lower molecular weight (figure 5 ), the intensity of peaks decreased and there was diffusion in the peaks. When the rabbits supplemented with omega-3 before IR exposure, the shift towards low molecular weight was decreased but the pattern didn't match the control. The intensity of peaks still was less than control.

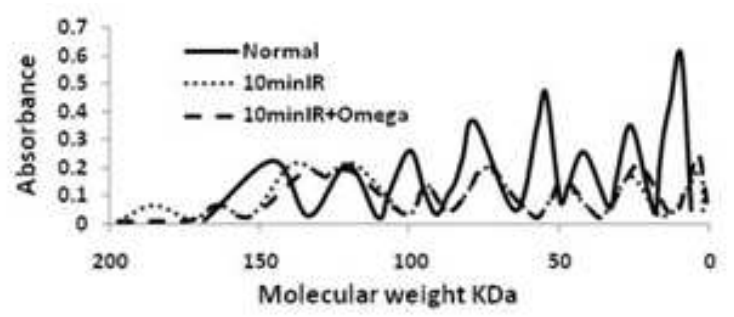

Figure (5) : Electrophor etic pattern of rat's retiana for normal and exposed to IR for $10 \mathrm{~min}$ with and withont onega-3.

When the rabbits exposed to IR for 20 minutes, the electrophoretic pattern dramatically changed with respect to control as shown in figure (6). All the peaks were diffused and its intensity significantly decreased. After omega-3 supplementation there was no recovery in the electrophoretic pattern.

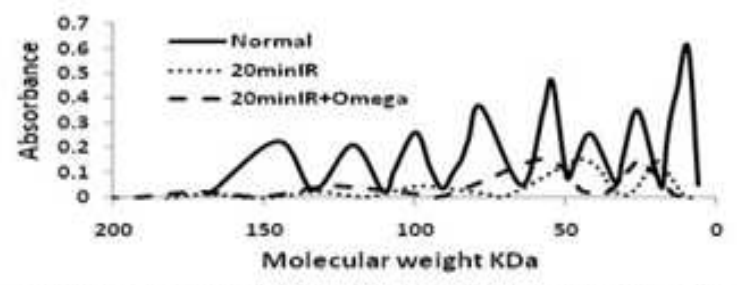

Figme (6) Electropher etic pattern of rar's retina for uermal and exposed to IR for $5 \mathrm{~min}$ with and withont omega-3.

\section{Discussion}

Exposure to IR radiation may cause forms of damage include scotoma, which is a loss of vision in aportion of the visual field resulting from damage to the retina where radiation is absorbed. Even low level in absorption can cause symptoms such as simple redness of the eye, but higher level can lead to swelling, hemorrhaging and lesion [23, 24].

In order to perform another quantitative analysis of photoreceptors response to retinal pigment epithelium and neurosensory retinal destruction, ERG was registered at different exposure time. The b-wave amplitude was strongly temperature dependent $[25,26]$. After 5 and 10 minutes of exposure to IR figure 2 showed ERG disturbances, b-wave amplitude reduced with increase exposure time and increase in b-wave latency b-wave reduction would result from greater defect in signal transmission between photoreceptor and bipolar cells, temperature can affect the functional properties of photoreceptors in a variety of way, it may directly alter the composition and characteristics of the membranes, the cytoplasm and visual molecules, it may affect certain features of the cellular metabolism, or it can exert an effect on the hormonal system of the animal, which in turn, may influence the adaptation state and thus determine visual sensitivity [27] nevertheless, the complete RPE disintegration and subsequent acute photoreceptor degeneration observed after 20 minutes exposure. Figures (6) and (7) resulted in total suppression of scotopic retinal function. This indicates advanced functional damage to the whole retinal area.

Evidence by SDS-PAGE, after exposure times ( 5 and 10 minutes ) are given in figures (4) and (5) it is clear that there were change in the molecular weight, electrophoretic mobility and intensity of different peaks resulting different retinal proteins fractions, after 20 minutes exposure, figure (3) showed disappearance of major retinal protein pattern, there is a lack of expression of protein or expression of new stressed protein these results attributed to IR radiation may cause at diverse biological effects of thermal origin [7]. There is a relationship between exposure time and thermal effect [28-30]. Irreversible thermal damage in the retina typically occurs only after the ambient temperature in the retina raised at least $10^{\circ} \mathrm{C}$ [31]. Depending on the extent of damage induced by the rise in thermal energy, cells may undergo apoptosis secondary to lower level thermal damage $\left(55-58^{\circ} \mathrm{C}\right)$, apoptosis and necrosis For more severe levels of thermal damage $\left(60-68^{\circ} \mathrm{C}\right)$. In contrast to apoptosis necrosis is usually induced by massive physical or chemical injury moreover it affect groups of cells and is accompanied by inflammation, and immediate cell death secondary to more severe thermal exposure $\left(72^{\circ} \mathrm{C}\right.$ or greater) on cellular and molecular level, increase in temperature cause denaturation of proteins, loss of molecular tertiary structure, and fluidization of membranes [32-34]. Infrared rays that are transmitted through the ocular media to the retina are absorbed by the pigment epithelium (PE) of the retina injury occurs in the neural layers through indirect heating. The effect of the infrared on the retina causes enzymes to denature, which play important roles in the process of energy production of cells, additionally the protein translation machinery which may affects on the membrane structure [10]. Heat stress by 
elevation of temperature induce the hsp70 (heat shock protein) in cultured retina and (hsp70) mRNA species in vivo retina [35-37]. It is expected that the production of such a stress protein contribute to changing membrane structure. This structural alteration in the retina and RPE may change the transport mechanism between them for rhodopsin regeneration resulting in reduction of threshold and the amplitude of ERG parameters [38].

No retinal changes have been shown in ERG record and electrophoretic pattern after 5 minutes exposure in rabbits supplemented with n-3 fatty acid. After 10 minutes rabbits that were given n-3 fatty acid showed a slower progression of retinal lesions. The results demonstrated an obvious benefit of long chain n-3 fatty acid intake. The beneficial effects of n-3 fatty acids in various physiological and pathological situations are well-documented [39]. The n-3 fatty acids act as an energy source as well as an important unique cell component, especially in cellular membranes. DHA is particularly rich in retinal photoreceptor outer segments [39]. The highest concentrations in the body for DHA per unit area are found in the photoreceptor disk membranes and the overall percentage of DHA can reach $50 \%$ of total retinal fatty acids [40, 41]. The unique fatty acid composition in retinal photoreceptor outer segment disk membranes is essential for maintaining a healthy retina. DHA affects membrane function by altering permeability, membrane order, thickness, lipid phase properties, and the activation of membrane-bound proteins [17].The special structure of DHA, the position of the first unsaturated bond at the n-3 fatty acids (between $\Delta-20$ and $\Delta-19$ ) carbon, provides an advantage in efficiency of membrane dynamics over that observed in an otherwise structurally identical fatty acid with the first double bond at the $n-6$ carbon [41]. The n-3 fatty acids and their derivatives play an extensive role in numerous biological processes, such as inflammatory cascades, apoptosis, and neuroprotection [17]. These are related to direct actions on plasma membranes, altered inflammatory response and control of gene expression. We focus on the role of n-3 fatty acids in inflammation because the role of inflammation after IR exposure is evident. We focused on the arachidonic acid metabolism pathway because it interacts with n-3 fatty acids metabolism in various ways such as plasma membranes [17]. Neuronal membranes are composed of high levels of PUFA, especially DHA and arachidonic acids [39]. Arachidonic acid (C20:4n-6) is the substrate for the synthesis of a range of biologically active compounds (eicosanoids) including prostaglandins, thromboxanes, and leukotrienes. These compounds can act as mediators in leukocyte chemotaxis and inflammatory cytokine production. When fish oil (high n-3 fatty acids) is provided, EPA is incorporated into cell membrane phospholipids at the expense of arachidonic acid, leading to less substrate available for eicosanoid synthesis[42], high n-3 fatty acids decreased the production of inflammatory eicosanoids (PGE2 and LTB4), which was correlated with amelioration of retinal lesions. High levels of n-3 fatty acids had lesion reversion may be via a shunted arachidonic acid metabolism that resulted in decreased pro-inflammatory derivatives (prostaglandin E2 and leukotriene B4) and an increased anti-inflammatory derivative (prostaglandin D2). No significant difference in the subgroups without or with n-3 fatty acid supplementation after 20-minutes of exposure. As a result extreme exposure of the eye, may cause dominate retinal burns and regenerative capabilities of the retina are very limited, therefore any retinal damage results in severe loss of visual acuteness [7].

\section{References}

[1] International Commission on Illumination (1987) International Lighting Vocabulary. CIE 17.4

[2] Ham W.T., Mueller H.A., and Sliney, D.H. Retinal sensitivity to damage from short wavelength light. Nature 260, 1976; pp153-155.

[3] Chen J.C, Lee L.R. "Solar retinopathy and associated optical coherence tomography findings." Clin Exp Optom. Nov;87(6):2004;pp390-3.

[4] Pitts D.G., Cullen A.P. and Dayhaw-Barker P. (1980) "Determination of ocular threshold levels for infrared radiation cataractogenesis" US Dept. Health and Human Sciences, National Institute for Occupational Safety and Health Publications 80-121.

[5] Sliney D.H and Freasier B.C Evaluation of Optical Radiation Hazards. Applied Optics 12, 1973; 1-2X

[6] Lydahl E. Infrared radiation and Cataract Acta Ophthalmol Suppl 1984;166:1-63.

[7] Kourkoumelis, N. and Tzaphlidou, M. Eye safety related to near infrared radiation exposure to biometric devices. The Scientific World JOURNAL 11, 2011; pp 520-528.

[8] Pitts D.G. and Cullen A.P. Determination of infrared radiation levels for acute ocular cataractogenesis. Albrecht Von Graefes Arch. Klin. Exp. Ophthalmol. 217,1981; 285-297.

[9] Allen R.G. Polhamus G.D. Ocular thermal injury from intense light In: Wolbarasht, M.L., ed, Laser application in medicine and biology. New : Plenum Press ; 1989.

[10] Voke J. Radiation effects on the eye, part 1: infrared radiation effects on ocular tissue. Optom. Today 9,1999;pp 22-28.

[11] Ham W. et al "Sensitivity of the retina to radiation damage as a function of wavelength". Photochem. And Photobiol. 29: 1979;pp 735-743

[12] Scott JA. The computtion of temperature rises in the human eye induced by infrared radiation. Phys Med Biol. 33: 1988;pp243-57.

[13] Kojima M, Okuno T, Miyakoshi M, Sasaki K, Takahashi N. Environmental temperature and cataract progression in experimental rat cataract models. Dev Ophthalmol. 35: 2002;pp125-34.

[14] International Commission on Non-Ionizing Radiation Protection Guidelines of limits of exposure to broad band incoherent optical radiation $(0.38$ to $3 \mu \mathrm{m})$. Health Phys. 73,1997 ; pp539-554. 
[15] Kang ZB, Ge Y, Chen Z, Cluette-Brown J, Laposata M, Leaf A, Kang JX. Adenoviral gene transfer of Caenorhabditis elegans $n-3$ fatty acid desaturase optimizes fatty acid composition in mammalian cells. Proc Natl Acad Sci USA.;98: 2001;pp4050-4054.

[16] Bazan NG. Omega-3 fatty acids, pro-inflammatory signaling and neuroprotection. Curr Opin Clin Nutr Metab Care. 10: 2007;pp136-141.

[17] Sangiovanni JP, Chew EY. The role of omega-3 long-chain polyunsaturated fatty acids in health and disease of the retina. Prog Retin Eye Res. 24: 2005;pp87-138.

[18] Rotstein NP, Politi LE, German OL, Girotti R. Protective effect of docosahexaenoic acid on oxidative stress-induced apoptosis of retina photoreceptors. Invest Ophthalmol Vis Sci. 44: 2003;pp2252-2259.

[19] Birch EE, Birch DG, Hoffman DR, Uauy R. Dietary essential fatty acid supply and visual acuity development. Invest Ophthalmol Vis Sci. 33: 1992;pp3242-3253.

[20] Rotstein NP, Aveldano MI, Barrantes FJ, Politi LE. Docosahexaenoic acid is required for the survival of rat retinal photoreceptors in vitro. J Neurochem. 66: 1996;pp1851-1859.

[21] Ronald TK, Ronald MB, Bernard B. Breakdown of the bloodaqueous barrier in the rabbit eye by infrared radiation. Invest Ophthalmol Vis Sci 30: 1989;pp717-22.

[22] Laemmli UK. Cleavage of structural proteins during assembly of the head of bacteriophage T4. Nature 1970;227:680 5 .

[23] D. Virgil Alfaro III, Peter E. Liggett, William F. Mieler, Hugo Quiroz-Mercado, Rama D. Jager,and Yasuo Tano,Age-Related Macular Degeneration: A Comprehensive Textbook , (Philadelphia,Lippincott Williams \& Wilkins, (2006).

[24] Roger L. Brauer, Safety and Health for Engineers: Second Edition, (John Wiley \& Sons, Inc., 2006

[25] Adolph, A. R. Temporal transfer and nonlinearity properties of turtle ERG: Tuning by temperature, pharmacology, and light intensity.Vision Research 25:1985;pp 483-492.

[26] Armington, J. C. and Adolph, A. R.Temperature effects on the electroretinogram of the isolated carp retina. Acta Ophthalmologica 62: 1984;pp498-509

[27] Kleinholz, L. H. () Hormonal regulation of retinal pigment migration in crustaceans. The Functional Organization of the Compound Eye1966;pp89-101
[28] Crochet JJ, Gnyawali SC, Chen Y, Lemley EC, Wang LV, Chen WR. Temperature distribution in selective laser-tissue interaction. J Biomed Opt. 2006;11 (3:34031.

[29] Darrigol O. A simplified genesis of quantum mechanics. Stud Hist Philos Mod Phys. 40: 2009;pp151-166.

[30] Fu JWZG, Wan K, Lin LY. A possible model: photothermal excitation via an excited state in the Si:Pd level. J Appl Phys. $64,10: 1988 ; p p 5266-5269$.

[31] Youssef P N, Sheibani N, Albert D M. Retinal light toxicity Eye (Lond) January; 25(1): 2011;pp1-14

[32] Birngruber R, Gabel VP, Hillenkamp F. Experimental studies of laser thermal retinal injury. Health Phys. 1983;44 (5:519-531.

[33] Birngruber R, Hillenkamp F, Gabel VP. Theoretical investigations of laser thermal retinal injury. Health Phys. 1985;48 (6:781-796.

[34] Henriques FC. Studies of thermal injury. Arch Pathol. 43: 1947;pp489-502.

[35] Masing, T. E., Rush S. J. and Brown I. R. Induction of a heat shockgene (hsp70) in rabbit retinal ganglion cells detected by in situ hybridization with plastic-embedded tissue.Neurochemical Research 15, 1990;pp1229-1235.

[36] Tytell M., Barbe, M. F. and Brown I. R. Induction of heat shock (stress) protein 70 and its mRNA in the normal and light-damaged rat retina after whole body hyperthermia. J. Neurosci. Res. 38 , 1994;pp 19-31.

[37] Tytell M. Heat Shock Proteins in the Retina and Optic Nerve.Heat Shock Proteins in the Nervous System 1994; (Chapter 4) 83-100

[38] ChonY.S. and KimY.Y.Modification of Retinal Function by Hypothermia and Hyperthermia Journal of Photoscience(2000), Vol. 7(4), pp. 161-167

[39] Dyall SC, Michael-Titus AT. Neurological benefits of omega-3 fatty acids. Neuromolecular Med. 10: 2008;pp219-235.

[40] Gawrisch K, Eldho NV, Holte LL. The structure of DHA in phospholipid membranes. Lipids.;38: 2003pp445-452.

[41] Mitchell DC, Niu SL, Litman BJ. DHA-rich phospholipids optimize G-Protein-coupled signaling. J Pediatr. 143: 2003;ppS80-S86.

[42] Calder PC. Long-chain n-3 fatty acids and inflammation: potential application in surgical and trauma patients. Braz $\mathrm{J}$ Med Biol Res. 36: 2003;pp433-446. 